DOI: $10.17516 / 1997-1397-2020-13-4-414-421$

УДК 512.7

\title{
Algebraic Geometry over Heyting Algebras
}

\author{
Mahdiyeh Nouri* \\ Faculty of Mathematical Sciences \\ University of Tabriz \\ Tabriz, Iran
}

Received 06.04.2020, received in revised form 25.04.2020, accepted 16.05.2020

\begin{abstract}
In this article, we study the algebraic geometry over Heyting algebras and we investigate the properties of being equationally Noetherian and $q_{\omega}$-compact over such algebras.

Keywords: universal algebraic geometry, systems of equations, radicals, Zariski topology, Heyting algebras, equationally Noetherian algebras, $q_{\omega}$-compact algebras.
\end{abstract}

Citation: M. Nouri, Algebraic Geometry over Heyting Algebras, J. Sib. Fed. Univ. Math. Phys., 2020, 13(4), 414-421. DOI: 10.17516/1997-1397-2020-13-4-414-421.

Universal algebraic geometry is a new area of modern algebra, whose subject is basically the study of equations over an arbitrary algebraic structure $A$ (see [11]). In the classical algebraic geometry $A$ of type $\mathcal{L}$ is a field. Many articles already published about algebraic geometry over groups, see $[1,8,16]$, and [10]. O. Kharlampovich and A. Miyasnikov developed algebraic geometry over free groups to give affirmative answer for an old problem of Alfred Tarski concerning elementary theory of free groups (see [7] and also [15] for the independent solution of Z. Sela). Also in [9], a problem of Tarski about decidablity of the elementary theory of free groups is solved. Algebraic geometry over algebraic structures (universal algebraic geometry) is also developed for algebras other than groups. A systematic study of universal algebraic geometry is done in a series of articles by V. Remeslennikov, A. Myasnikov and E. Daniyarova in [2-4], and [5].

The notations of the present paper are standard and can be find in [2] or [11]. Our main aim in this article is to deal with the equational conditions in the universal algebraic geometry over Heyting algebras, i.e. different conditions relating systems of equations especially conditions about systems and sub-systems of equations over algebras. The main examples of such conditions are equational noetherian property and $q_{\omega}$-compactness. We begin with a review of basic concepts of universal algebraic geometry and we describe the properties of being being equational noetherian, $q_{\omega}$-compact. We will show that only finite Heyting algebras have these properties.

\section{Basic notions}

We need to give a brief introduction of universal algebraic geometry. Our notations here are almost the same as in the above mentioned papers, especially [11].

We begin with an algebraic language $\mathcal{L}$ and an arbitrary algebra $A$ type $\mathcal{L}$ and then we extended the language by adding new constant symbols $a \in A$. This extended language will be denoted by $\mathcal{L}(A)$. An algebra $B$ of type $\mathcal{L}(A)$ is called $A$-algebra, if the map $a \mapsto a^{B}$ is an embedding of $A$ in $B$. In this notation, $a^{B}$ denotes the interpretation of the constant symbol $a$ in $B$.

Suppose that $X=\left\{x_{1}, \ldots, x_{n}\right\}$ is a finite set of variables. We denote the term algebra in the language $\mathcal{L}$ and variables from $X$ by $T_{\mathcal{L}}(X)$, and similarly the term algebra in the extended

*mahtopology@gmail.com

(C) Siberian Federal University. All rights reserved 
language $\mathcal{L}(A)$ will denoted by $T_{\mathcal{L}(A)}(X)$. For the sake of simplicity, we define our notions in the coefficient free frame, i.e. in the language $\mathcal{L}$ and then we can extend all the definitions to the language $\mathcal{L}(A)$.

An equation is a pair $(p, q)$ of the elements of the term algebra $T_{\mathcal{L}}(X)$. In many cases, we assume that such an equation is the same as the atomic formula $p\left(x_{1}, \ldots, x_{n}\right) \approx q\left(x_{1}, \ldots, x_{n}\right)$ or $p \approx q$ in short.

Any set of equations is called a system of equations in the language $\mathcal{L}$. A system $S$ is called consistent over an algebra $A$, if there is an element $\left(a_{1}, \ldots, a_{n}\right) \in A^{n}$ such that for all equations $(p \approx q) \in S$, the equality

$$
p^{A}\left(a_{1}, \ldots, a_{n}\right)=q^{A}\left(a_{1}, \ldots, a_{n}\right)
$$

holds. Otherwise, we say that $S$ is in-consistent over $A$. Note that, $p^{A}$ and $q^{A}$ are the corresponding term functions on $A^{n}$. A system of equations $S$ is called an ideal, if it corresponds to a congruence on $T_{\mathcal{L}}(X)$. For an arbitrary system of equations $S$, the ideal generated by $S$, is the smallest congruence containing $S$ and it is denoted by $[S]$.

For an algebra $A$ of type $\mathcal{L}$, an element $\left(a_{1}, \ldots, a_{n}\right) \in A^{n}$ will be denoted by $\bar{a}$, sometimes. Let $S$ be a system of equations. Then the set

$$
V_{A}(S)=\left\{\bar{a} \in A^{n}: \forall(p \approx q) \in S, p^{A}(\bar{a})=q^{A}(\bar{a})\right\}
$$

is called an algebraic set. It is clear that for any non-empty family $\left\{S_{i}\right\}_{i \in I}$, we have

$$
V_{A}\left(\bigcup_{i \in I} S_{i}\right)=\bigcap_{i \in I} V_{A}\left(S_{i}\right)
$$

It is possible to define a topology on $A^{n}$ using algebraic sets as elements of subbasis: define a closed set in $A^{n}$ to be an arbitrary intersections of finite unions of algebraic sets. Therefore, we obtain a topology on $A^{n}$, which is called Zariski topology.

For any set $Y \subseteq A^{n}$, we define

$$
\operatorname{Rad}(Y)=\left\{(p, q): \forall \bar{a} \in Y, p^{A}(\bar{a})=q^{A}(\bar{a})\right\} .
$$

It is easy to see that $\operatorname{Rad}(Y)$ is an ideal in the term algebra. Any ideal of this type is called an $A$-radical ideal or a radical ideal for short. Note that any ideal in the term algebra is in fact a radical ideal. To see the reason, just note that for any ideal $R$ in the term algebra $T_{\mathcal{L}}(X)$, if we consider the algebra $B(R)=T_{\mathcal{L}}(X) / R$, then $\operatorname{Rad}_{B(R)}(R)=R$.

It is easy to see that a set $Y$ is algebraic if and only if $V_{A}(\operatorname{Rad}(Y))=Y$. In the general case, we have $V_{A}(\operatorname{Rad}(Y))=Y^{a c}($ see [3]). The coordinate algebra of a set $Y$ is the quotient algebra

$$
\Gamma(Y)=\frac{T_{\mathcal{L}}(X)}{\operatorname{Rad}(Y)} .
$$

An arbitrary element of $\Gamma(Y)$ is denoted by $[p]_{Y}$. We define a function $p^{Y}: Y \rightarrow A$ by the rule

$$
p^{Y}(\bar{a})=p^{A}\left(a_{1}, \ldots, a_{n}\right),
$$

which is a term function on $Y$, for all $a_{1}, \ldots, a_{n} \in A$. The set of all such functions will be denoted by $T(Y)$ and it is naturally an algebra of type $\mathcal{L}$. It is easy to see that the map $[p]_{Y} \mapsto p^{Y}$ is a well-defined isomorphism. So, we have $\Gamma(Y) \cong T(Y)$.

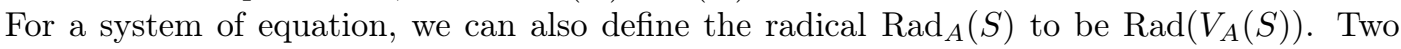
systems $S$ and $S^{\prime}$ are called equivalent over $A$, if they have the same set of solutions in $A$, i.e. $V_{A}(S)=V_{A}\left(S^{\prime}\right)$. So, clearly $\operatorname{Rad}_{A}(S)$ is the largest system which is equivalent to $S$. Note that $[S] \subseteq \operatorname{Rad}_{A}(S)$. 
One of the major problems of the universal algebraic geometry is to determine the structures of algebras which appear as the coordinate algebras. There are many necessary and sufficient conditions for an algebra to be a coordinate algebra and we will give a summary of such results in the Subsection 2.4.

In this article, we are dealing with equational conditions on algebras. The first and maybe the most important condition of this type can be formulated as follows.

Definition 1. An algebra $A$ is called equational Noetherian, if for any system of equations $S$, there exists a finite subsystem $S_{0} \subseteq S$, which is equivalent to $S$ over $A$, i.e. $V_{A}(S)=V_{A}\left(S_{0}\right)$.

If an $A$-algebra is equational Noetherian in the language $\mathcal{L}(A)$, then we call it $A$-equational Noetherian. Many examples of equational Noetherian algebras are introduced in [3]. Among them are Noetherian rings and linear groups over Noetherian rings as well as free groups. In [3], it is proved that the next four assertions are equivalent:

i- An algebra $A$ is equational Noetherian.

ii- For any system $S$, there exists a finite $S_{0} \subseteq[S]$, such that $V_{A}(S)=V_{A}\left(S_{0}\right)$.

iii- For any n, the Zariski topology on $A^{n}$ is Noetherian, i.e. any descending chain of closed subsets terminates.

iv- Any chain of coordinate algebras and epimorphisims

$$
\Gamma\left(Y_{1}\right) \rightarrow \Gamma\left(Y_{2}\right) \rightarrow \Gamma\left(Y_{3}\right) \rightarrow \cdots
$$

terminates.

So, in the case of equational Noetherian algebras, any closed set in $A^{n}$ is equal to a minimal finite union of irreducible algebraic sets which is unique up to a permutation. Note that a set is called irreducible, if it has no proper finite covering consisting of closed sets. The following theorem is proved in [3].

Theorem 1. Let $A$ be an equational Noetherian algebra. Then the following algebras are also equational Noetherian:

$i$ - any subalgebra and filter-power of $A$.

ii- any coordinate algebra over A.

iii- any fully residually A-algebra.

$i v$ - any algebra belonging to the quasi-variety generated by A.

$v$ - any algebra universally equivalent to $A$.

vi- any limit algebra over $A$.

vii- any finitely generated algebra defined by a complete atomic type in the universal theory of $A$ or in the set of quasi-identities of $A$.

The most important theorem for equationally Noetherian algebras is called Unification Theorem. It describes the structure of coordinate algebras over equationally Noetherian algebras. For a proof of this theorem (see [2]). 
Theorem 2. Let $A$ and $\Gamma$ be algebras in a language $\mathcal{L}$. Suppose $A$ is equational Noetherian and $\Gamma$ is finitely generated. Then the following assertions are equivalent.

$i-\Gamma$ is the coordinate algebra of some irreducible algebraic set over $A$.

ii- $\Gamma$ is a fully residually A-algebra. This means that for any finite subset $C \subseteq \Gamma$, there exists a homomorphism $\alpha: \Gamma \rightarrow A$, such that the restriction of $\alpha$ to $C$ is injective.

iii- $\Gamma$ embeds into some ultra-power of $A$.

iv- $\Gamma$ belongs to the universal closure of $A$, i.e. $T h_{\forall}(A) \subseteq T h_{\forall}(\Gamma)$.

$v-\Gamma$ is a limit algebra over $A$.

$v i$ - $\Gamma$ is defined by a complete type in $T h_{\forall}(A)$.

There are similar theorems for the cases where $A$ is $q_{\omega}$-compact. Note that an algebra $A$ is called $q_{\omega}$-compact, if for any system $S$ and any equation $p \approx q$, the condition $V_{A}(S) \subseteq V_{A}(p \approx q)$ implies that $V_{A}\left(S_{0}\right) \subseteq V_{A}(p \approx q)$ for some finite $S_{0} \subseteq S$. Clearly, every equationally Noetherian algebra is $q_{\omega}$-compact.

A. Shevlyakov studied algebraic geometry over Boolean algebras, [17]. He obtained necessary and sufficient condition for a Boolean algebra to be equationally Noetherian or to be $q_{\omega}$-compact. Let $B$ be a Boolean algebra and $C$ be a subalgebra of $B$. Then we can consider $B$ as a $C$-algebra. Shevlyakov proved that $B$ is $C$-equationally Noetherian, if and only if $C$ is finite. Consequently only finite Boolean algebras are equationally Noetherian in the case of Diophantine algebraic geometry. He also obtained necessary and sufficient conditions for the $C$-Boolean algebra $B$ to be $q_{\omega}$-compact. To explain it, we need to define $E_{k}$-systems: a system of $C$-equations $S$ is called $E_{k}$ system over $B$, if $V_{B}(S)$ has $k$ elements, but for any finite subsystem $S^{\prime} \mp S$, the algebraic set $V_{B}\left(S^{\prime}\right)$ is infinite. It is proved that $B$ is $q_{\omega}$-compact as a $C$-algebra, if and only if there are no any $E_{0}$ and $E_{1}$-systems over $B$.

In this article, we are dealing with the case of Heyting algebras, which are natural generalizations of Boolean algebras.

\section{Algebraic geometry over Heyting algebras}

Heyting algebras for propositional intuitionistic logic are the same as Boolean algebras for classical propositional logic. Note that in intuitionistic logic, truth is equivalent to provability. Since by the incompleteness theorem of Godel, there are sentences $\alpha$ in the language of arithmetic such that $\alpha \vee \neg \alpha$ is not provable, so in the case of intuitionistic logic, Boolean algebras are not useful and one must employ the more general frame of Heyting algebras. A Heyting algebra is a bounded lattice $H$, such that for all $a, b \in H$, there exists a maximum element $x$ with the property $a \wedge x \leqslant b$. Let's denote that element $x$ by $a \rightarrow b$. Then, one can see that the following identities are hold

1) $a \rightarrow a=1$.

2) $a \wedge(a \rightarrow b)=a \wedge b$.

3) $b \wedge(a \rightarrow b)=b$.

4) $a \rightarrow(b \wedge c)=(a \rightarrow b) \wedge(a \rightarrow c)$. 
So, let $\mathcal{L}=(\wedge, \vee, \rightarrow, 1,0)$ be the language of bounded lattices extended by adding a new binary symbol $\rightarrow$. Then the variety of Heyting algebras is just the variety axiomatized by the identities of bounded lattices plus the above four new identities. Let $\neg a=a \rightarrow 0$. It can be shown that in any Heyting algebra, we have only one of the De Morgan's laws, namely $\neg(a \vee b)=\neg a \wedge \neg b$, but the other law $\neg(a \wedge b)=\neg a \vee \neg b$ is not valid, despite the case of Boolean algebras. It is also true that $\neg \neg \neg a=\neg a$ and $\neg a \vee \neg \neg a=1$. Recall that a complete Heyting algebra is a Heyting algebra which is also a complete lattice.

An element $a$ in a Heyting algebras $H$ is called regular, if $\neg \neg a=a$. Clearly, both 0 and 1 are regular. Let $H_{\text {reg }}$ be the set of all regular elements of $H$. It is easy to see that $H_{\text {reg }}=\neg H$, the set of all negated elements of $H$. This set is not a Heyting subalgebra in general, but it is a Boolean algebra bey the following operations

1) $a \wedge_{\text {reg }} b=a \wedge b$.

2) $\neg$ reg $a=\neg a$.

3) $a \vee_{\text {reg }} b=\neg(\neg a \wedge \neg b)$.

We will use the notation $\mathcal{L}_{\text {reg }}$ for the Boolean language $\left(\wedge, \vee_{\text {reg }}, \neg, 0,1\right)$. This will help us to apply results of [17] for Heyting algebras.

Note that, despite Boolean algebras, the free Heyting algebra $F_{H}(X)$ is always infinite for any non-empty set $X$. For example, if $X=\{x\}$, then the free Heyting algebra over $X$ consists of the following elements

$$
0, x, \neg x, \neg \neg x, x \vee \neg x, \neg x \vee \neg \neg x, \neg \neg x \rightarrow x,(\neg \neg x \rightarrow x) \rightarrow(x \vee \neg x), \ldots
$$

We focus on the case of equations with coefficients inside $H$ (Diophantine Geometry). It is known that free groups are equationally Noetherian. Free Boolean algebras of finite rank are also equationally Noetherian since they are finite. We show that no non-trivial Heyting algebra is equationally Noetherian.

Proposition 1. Let $X$ be a non-empty set. Then the free Heyting algebra $F_{H}(X)$ is not equationally Noetherian.

Proof. It is enough to consider the case $X=\{p\}$, because subalgebras of equationally Noetherian algebras are again equationally Noetherian. Consider the following infinite chain

$$
p<\neg \neg p<\neg p \vee \neg \neg p<\cdots .
$$

Let $S$ be the system $\{x \geqslant p, x \geqslant \neg \neg p, x \geqslant \neg p \vee \neg \neg p, \ldots\}$. It is obvious that $V_{F}(S)=\{1\}$. But, since the above chain is infinite, so for every finite $S_{0} \subseteq S$, there are infinitely many elements in $V_{F}\left(S_{0}\right)$. Hence $V_{F}(S) \neq V_{F}\left(S_{0}\right)$. This shows that $F=F_{H}(X)$ is not equationally Noetherian.

Note that in the same time the above argument shows that non-trivial free Heyting algebras are not $q_{\omega}$-compact. This is true because we have $V_{F}(S) \subseteq V_{F}(x \approx 1)$, but for any finite $S_{0} \subseteq S$, the algebraic set $V_{F}\left(S_{0}\right)$ is infinite.

We now, can use the same idea to prove that infinite Heyting algebras are not equationally Noetherian. It can be also applied for infinite complete Heyting algebras to prove that they are not $q_{\omega}$-compact.

Theorem 3. Let $H$ be a Heyting algebra and $K$ be a subalgebra, which is infinite. Then $H$ is not $K$-equationally Noetherian. 
Proof. For simplicity we discuss Diophantine case $(K=H)$. The idea of the proof is taken from a similar theorem for Boolean algebras (see [6]). Let

$$
b_{0}, b_{1}, b_{2}, \ldots
$$

be an infinite set of elements in $H$. Let $L_{0}=\{0,1\}$ and define $L_{n}$ by inductions as follows: if $L_{n-1}=\left\{a_{0}=0, a_{1}, \ldots, a_{n-1}, a_{n}=1\right\}$, and if $0 \leqslant i \leqslant n$, then define

$$
c_{i+1}=a_{i} \vee\left(a_{i+1} \wedge b_{n}\right) .
$$

For example, we have $L_{0}=\left\{a_{0}=0, a_{1}=1\right\}$. Then we compute

$$
c_{1}=a_{0} \vee\left(a_{1} \wedge b_{1}\right)=b_{1} .
$$

It is clear that $0 \leqslant b_{1} \leqslant 1$. Let $L_{1}=\left\{0, a_{1}, 1\right\}$ and rename its elements as $a_{0}=0, a_{1}=b_{1}, a_{2}=1$. Now, to find $L_{2}$, we compute

$$
c_{1}=a_{0} \vee\left(a_{1} \wedge b_{2}\right)=b_{1} \wedge b_{2},
$$

and

$$
c_{2}=a_{1} \vee\left(a_{2} \wedge b_{2}\right)=b_{1} \vee b_{2} .
$$

We have

$$
0 \leqslant b_{1} \wedge b_{2} \leqslant b_{1} \leqslant b_{1} \vee b_{2} \leqslant 1,
$$

so $L_{2}$ consists of the above elements. Again rename $a_{0}=0, a_{1}=b_{1} \wedge b_{2}, a_{2}=b_{1}, \ldots$, and continue this process. It is clear from the construction that

$$
L_{0} \subset L_{1} \subset L_{2} \subset \cdots,
$$

so the set $L=\cup_{n \geqslant 0} L_{n}$ is an infinite chain in $H$.

Now, we proved that there is an infinite chain $a_{0}<a_{1}<a_{2}<\cdots$ in $H$ so we can consider the following system

$$
S=\left\{x \geqslant a_{0}, x \geqslant a_{1}, x \geqslant a_{2}, \ldots\right\} .
$$

For any finite subsystem $S_{0}=\left\{x \geqslant a_{0}, x \geqslant a_{1}, x \geqslant a_{2}, \ldots, a_{n}\right\}$, we have $a_{n+1} \in V_{H}\left(S_{0}\right)$, while $a_{n+1}$ does not belong to $V_{H}(S)$. This proves that $H$ is not equationally Noetherian.

Note that if $H$ is complete, then in the above proof we can put $a=\sup _{i} a_{i}$. Then $V_{H}(S) \subseteq$ $V_{H}(x \geqslant a)$, but for any finite subset $S_{0}$, it is not true that $V_{H}\left(S_{0}\right) \subseteq V_{H}(x \geqslant a)$. This shows that if $H$ is a complete infinite Heyting algebra, then it is not $q_{\omega}$-compact. The next theorem concerns the relation between $q_{\omega}$-compactness of a Heyting algebra $H$ and $H_{\text {reg }}$.

Theorem 4. Let $H$ be $q_{\omega}$-compact. Then there is no $E_{0}$ and $E_{1}$-systems in the language $\mathcal{L}_{\text {reg }}$ over the Boolean algebra $H_{\text {reg }}$.

Proof. Assume that $S$ is an $\mathcal{L}_{\text {reg }}$-system of equations with $n$ indeterminate and denote $H_{\text {reg }}$ for simplicity by $R$. Let $V_{R}(S) \subseteq V_{R}(p \approx q)$, where $p \approx q$ is an $\mathcal{L}_{\text {reg-g}}$-equation. Note that $R^{n}$ is an algebraic set in $H^{n}$ because it is just the solution set of the system $\neg \neg x_{1} \approx x_{1}, \neg \neg x_{2} \approx$ $x_{2}, \ldots, \neg \neg x_{n} \approx x_{n}$. Hence we have

$$
V_{R}(S)=V_{H}\left(S+\neg \neg x_{1} \approx x_{1}, \ldots, \neg \neg x_{n} \approx x_{n}\right),
$$

and

$$
V_{R}(p \approx q)=V_{H}\left(p \approx q+\neg \neg x_{1} \approx x_{1}, \ldots, \neg \neg x_{n} \approx x_{n}\right) .
$$


This shows that

$$
V_{H}\left(S+\neg \neg x_{1} \approx x_{1}, \ldots, \neg \neg x_{n} \approx x_{n}\right) \subseteq V_{H}\left(p \approx q+\neg \neg x_{1} \approx x_{1}, \ldots, \neg \neg x_{n} \approx x_{n}\right),
$$

and hence

$$
V_{H}\left(S+\neg \neg x_{1} \approx x_{1}, \ldots, \neg \neg x_{n} \approx x_{n}\right) \subseteq V_{H}(p \approx q) .
$$

Since $H$ is assumed to be $q_{\omega}$-compact, so there is a finite subset $S_{0} \subseteq S+\neg \neg x_{1} \approx x_{1}, \ldots, \neg \neg x_{n} \approx$ $x_{n}$ such that $V_{H}\left(S_{0}\right) \subseteq V_{H}(p \approx q)$. Therefore, $V_{R}\left(S_{0}\right) \subseteq V_{R}(p \approx q)$. Let $S^{\prime}=S_{0} \backslash\left\{\neg \neg x_{1} \approx\right.$ $\left.x_{1}, \ldots, \neg \neg x_{n} \approx x_{n}\right\}$. Then we have

$$
V_{R}\left(S^{\prime}\right) \subseteq V_{R}\left(S_{0}\right) \subseteq V_{R}(p \approx q)
$$

and this shows that the Boolean algebra $R$ is $q_{\omega}$-compact in the Boolean language $\mathcal{L}_{\text {reg }}$. Now, we can apply the result of [17] to conclude that there are no $E_{0}$ and $E_{1}$-systems in the language $\mathcal{L}_{\text {reg }}$ over $H_{\text {reg }}$.

\section{References}

[1] G.Baumslag, A.Myasnikov, V.Remeslennikov, Algebraic geometry over groups I. Algebraic sets and ideal theory, J. Algebra, 219(1999), 16-79.

[2] E.Daniyarova, A.Myasnikov, V.Remeslennikov, Unification theorems in algebraic geometry, Algebra and Discrete Mathamatics, 1(2008), 80-112.

[3] E.Daniyarova, A.Myasnikov, V.Remeslennikov, Algebraic geometry over algebraic structures, II: Fundations, J. Math. Sci., 185(2012), no. 3, 389-416.

[4] E.Daniyarova, A.Myasnikov, V.Remeslennikov, Algebraic geometry over algebraic structures, III: Equationally noetherian property and compactness, South. Asian Bull. Math., 35(2011), no. 1, 35-68.

[5] E.Daniyarova, A.Myasnikov, V.Remeslennikov, Algebraic geometry over algebraic structures. IV: Equatinal domains and co-domains, Algebra and Logic, 49(2011), 483-508.

[6] S.Goncharov, Countable Boolean algebras and decidability, Cousultant Baurou, New York, 1997.

[7] O.Kharlampovich, A.Myasnikov, Tarski's problem about the elementary theory of free groups has a psitive solution, E.R.A. of AMS, 4(1998), 101-108.

[8] O.Kharlampovich, A.Myasnikov, Irreducible affine varieties over a free group. I: irreducibility of quadratic equations and Nullstellensatz, J. Algebra, 200(1998), no. 2, 472-516.

[9] O.Kharlampovich, A.Myasnikov, The elemntary theory of free non-abelian groups, J. Algebra, $\mathbf{3 0 2}(2006)$, 451-552.

[10] G.Makanin, Equations in a free group, Math. USSR-Izv., 21(1983), no. 3, 483-546.

[11] P.Modabberi, M.Shahryari, Compactness conditions in universal algebraic geometry, Algebra and Logic, 55(2016), no. 2, 146-172.

[12] P.Modabberi, M.Shahryari, On the equational Artinian algebras, Siberian Electronic Mathematical Reports, 13(2016), 875-881.

[13] B.Plotkin, Seven lectures in universal algebraic geometry, preprints, Arxiv, 2002. 
[14] A.Razborov, On systems of equations in free groups, Math. USSR-Izv., 25(1985), no. 1, $115-162$.

[15] Z.Sela, Diophantine geometry over groups IX: Envelopes and Imaginaries, arXiv:0909.0774, 2009 .

[16] M.Shahryari, A.Shevlyakov, Direct products, varieties, and compacness conditions, Groups Complexity Cryptology, 9(2017), no. 2, 159-166.

[17] A.Shevlyakov, Algebraic geometry over Boolean algebras in the language with constants, J. Math. Sciences, 206(2015), no. 6, 742-757.

\title{
Алгебраическая геометрия над гейтинговыми алгебрами
}

Махдия Нури

Факультет математических наук

Университет Тебриза

Тебриз, Иран

\begin{abstract}
Аннотация. В этой статье мы изучаем алгебраическую геометрию над гейтинговыми алгебрами и исследуем свойства быть уравновешенно нетеровыми и $q_{\omega}$-компактными над такими алгебрами.

Ключевые слова: универсальная алгебраическая геометрия, системы уравнений, радикалы, топология Зарисского, алгебры Гейтинга, нетеровы алгебры уравнений, $q_{\omega}$-компактные алгебры.
\end{abstract}

